\title{
Participación política de la juventud afrodescendiente en el Perú: recambio generacional y derechos humanos
}

\author{
Political participation of African descent youth in Peru: Generational change and \\ human rights
}

Jorge Rafael Ramírez*

\begin{abstract}
Resumen:
Este artículo tiene como objetivo discutir la situación de pobreza y exclusión social que atraviesan la población afrodescendiente en el Perú, analizando el rol y las acciones del Estado peruano en la implementación de políticas públicas y el respeto a los derechos humanos de la población afroperuana a medida de generar procesos de inclusión social y desarrollo con identidad. Se resaltarán para ese sentido, y a manera de recambio generacional al Movimiento Afroperuano, algunos mecanismos de organización y participación política de las y los jóvenes afrodescendientes en el Perú para la incidencia de políticas públicas de inclusión social a fin de contribuir al respeto de los derechos humanos, políticos y al desarrollo étnico-racial. Como metodología se realizaron estudios bibliográficos documentales y entrevistas a las y los dirigentes de Ashanti Perú la Red Peruana de Jóvenes Afrodescendientes colocando la experiencia exitosa de incidencia de la Agenda de la Juventud Afroperuana y la Escuela de Formación de Jóvenes Lideres Afrodescendientes presentando demandas y concepciones que permiten alimentar el debate sobre la necesidad de un recambio generacional al Movimiento Afrodescendiente y la generación de políticas de acciones afirmativas.
\end{abstract}

Palabras-clave: Derechos humanos; Inclusión social; Afrodescendientes; Jóvenes afroperuanos; Participación política.

\begin{abstract}
:
This article aims to discuss the situation of poverty and social exclusion that cross the black population in Peru, analyzing the role and actions of the Peruvian State in the implementation of public policies and respect for human rights of Afro-Peruvian population as to generate processes of social inclusion and development with identity. They will be highlighted to that effect, and by way of the Afro-Peruvian Movement generational change, some mechanisms and political organization and participation of young people of African descent in Peru for the incidence of public policies for social inclusion to contribute to respect for human rights, political and ethnic-racial development. The methodology documentaries bibliographic studies and interviews with key leaders of Ashanti Peru Peruvian Youth Network of African Descent were performed by placing the successful experience of incidence of Agenda Afroperuana Youth and Training School for Young Leaders of African Descent filing lawsuits and conceptions allow fuel the debate about the need for a generational change to the Afro-descendant movement and the generation of affirmative action policies.
\end{abstract}

\footnotetext{
* Assistente Social. Cursando mestrado no Programa de Serviço Social e Política Social da Universidade Estadual de Londrina UEL. E-mail: joramirez4@gmail.com
} 
Key words: Human rights; Social inclusion; African descent; Afro-Peruvian youth; Political participation.

\section{Introducción}

La población afrodescendiente en el Perú es un sector de la población en general que aún continúa atravesando por profundos problemas sociales, económicos y políticos. Miles de afroperuanos y afroperuanas siguen sufriendo las consecuencias del racismo y la vulneración de sus derechos, manteniendo como herencia las consecuencias de la esclavitud y el colonialismo.

Según Marx (2001, p. 108):

La esclavitud directa es la base de la industria burguesa. Sin esclavitud no habría algodón; sin algodón no habría industria moderna. La esclavitud ha dado valor a las colonias, las colonias han creado el comercio universal, el comercio universal es la condición necesaria de la gran industria. Por tanto, la esclavitud es una categoría económica de la más alta importancia.

El Estado peruano como parte del bloque de países capitalistas de América del Sur ha mostrado un poco interés por generar medidas y políticas que puedan contribuir a erradicar los rezagos de esclavitud y colonialismo hacia la población afroperuana para promover justicia social y desarrollo con identidad.

En un país con grupos poblacionales mayoritariamente andinos y amazónicos, el Estado peruano ha reconocido la existencia de estas poblaciones1 y evidencia tener el compromiso de promover su desarrollo e inclusión social, pero estas acciones excluyen e invisibilizan a la población afrodescendiente, recayendo en que su visibilidad ante el Estado, la defensa de sus derechos humanos y el desarrollo comunitario sea trabajo del movimiento afroperuano y sus jóvenes.

Tomando como base el proceso de organización y lucha del movimiento afroperuano, el presente artículo busca discutir los procesos de invisibilidad, el estado de los derechos humanos y la exclusión que atraviesa la población afrodescendiente en el Perú, además de explorar el proceso organizativo del movimiento juvenil afroperuano

\footnotetext{
${ }^{1}$ Constitución Política del Perú (COMITÉ DE LAS NACIONES UNIDAS PARA LA ELIMINACIÓN DE LA DISCRIMINACIÓN RACIAL, 2014).
} 
tomando la experiencia de Ashanti Perú la Red Peruana de Jóvenes Afrodescendientes en el ámbito de los derechos humanos y la participación política.

Este artículo es referencia de una investigación ya concluida, utilizando una metodología bibliográfica documental realizada en base a una amplia investigación de documentos y autores que discuten sobre los obstáculos y desafíos de la población afrodescendiente en el Perú, queriendo responder algunas cuestiones como: ¿El Estado peruano influye en la vulneración de los derechos humanos y la invisibilidad de las poblaciones afroperuanas? Y ¿Cuáles son los mecanismos de organización y participación política de las juventudes afroperuanas? A fin de abarcar y profundizar más sobre la realidad de esta población y la defensa de sus derechos.

Este texto contribuye a entender la importancia del recambio generacional del Movimiento Afroperuano en el empoderamiento de organizaciones juveniles afroperuanas para la continuidad de la lucha por los derechos humanos con estrategias y experiencias propias de las juventudes afroperuanas.

\section{El Estado Peruano y la población afroperuana: realidades y derechos pendientes}

Cuando pensamos en América Latina y el Caribe podemos evidenciar entre muchas características, un continente con un elevado índice de desigualdades, tanto sociales, económicas, políticas y culturales de las cuales una de las poblaciones que aún atraviesa procesos de exclusión e invisibilidad tanto por los Estados y la sociedad, es la población afrodescendiente.

Al hablar de la población afrodescendiente podemos abarcar un sector de la población a la cual en la actualidad se le debe tomar especial importancia en cuanto a generación de políticas públicas inclusivas y acciones afirmativas. Según la Organización de los Estados Americanos (2011, p. 5)

\footnotetext{
Existen de alrededor de $\mathbf{2 0 0}$ millones de habitantes afrodescendientes a lo largo y ancho de las Américas, que según lo han reconocido los órganos del Sistema Interamericano, continúan siendo víctimas del racismo, de la discriminación, y además, siguen siendo privados de algunos de sus derechos y necesidades básicas.
}

En el Perú las y los afrodescendientes corresponden aproximadamente al $10 \%$ de la población nacional ubicándose alrededor de toda la costa peruana, quienes a lo largo de los 
años han estado ocupando una posición subordinada en la estructura social, económica y política del Estado, lo cual ha originado un menor acceso a los beneficios del desarrollo socioeconómico. La situación de precariedad en la que viven muchas personas afroperuanas y afroperuanos sienta sus bases en el contexto de esclavitud, el colonialismo y de marginación social de la que han sido objeto a lo largo de la historia. Según Carlos Aguirre (2004, p. 2),

\footnotetext{
A pesar de los cambios ocurridos en el Perú en estos últimos doscientos años, la esclavitud no ha terminado. Pese a toda la importancia que fechas como 1804 y 1854 tienen como hitos decisivos en la lucha contra un régimen brutal de explotación como era la esclavitud de la población de origen africano, sus huellas siguen percibiéndose hoy en la supervivencia del racismo, la discriminación, y la marginalización de las poblaciones negras.
}

Si bien es cierto que a pesar de la crisis económica en muchos países de América Latina y Europa, la economía peruana se encuentra en un proceso de crecimiento, sin embargo, los niveles de pobreza, exclusión social y política en el país no han disminuido, el porcentaje de población pobre sigue siendo considerable y el índice de exclusión en espacios políticos y de toma de decisiones en vez de reducirse se ha incrementado, aumentando las diferencias entre quienes tienen mayor ingreso económico respecto a los que tienen menos ingresos, afectando de forma particular a las y los afroperuanos y especialmente a la juventud afroperuana.

El Estado peruano ha hecho muy poco frente a la situación de pobreza y ante las formas abiertas de racismo y prejuicios raciales, tampoco ha promovido una política social que iguale en oportunidades a la población afroperuana con el resto de la población del país. No existen políticas públicas de atención e incentivo que estimulen el progreso de las y los afroperuanos, ni tampoco se ha estimulado a aquellos que por sus habilidades personales podrían haber destacado y servido de ejemplo para otras personas.

A pesar de que en el proceso de reforma del Estado peruano y descentralización del país, se están fortaleciendo los sub niveles de gobiernos regionales y municipales y se ha incorporado a la ciudadanía en la corresponsabilidad de la gestión pública y de la promoción del desarrollo local, regional y nacional, sin embargo al reducirse el tamaño del Estado también se han reducido los espacios que promovían la inclusión de las comunidades nativas y las poblaciones afroperuanas, como ocurre en el caso del Instituto Nacional de los Pueblos Andinos Amazónicos y Afroperuanos - INDEPA que nació como un organismo público descentralizado que promovía políticas públicas para estas poblaciones 
y con rango Ministerial, institución que luego fue fusionada al Ministerio de la Mujer y Desarrollo Social - MIMDES², y actualmente ser parte del Ministerio de Cultura, eliminando su rango ministerial y convirtiéndose ahora en una simple oficina de difusión cultural, por lo que las poblaciones tanto indígenas como afroperuanas se encuentran en situación de exclusión por este órgano del Estado, con plena indiferencia de los acuerdos firmados en la Conferencia Mundial contra el Racismo, la Discriminación, la Xenofobia y otras formas conexas de Intolerancia de Durban en el año 2001 y del Convenio 169 de la OIT en el año 1989.

Cabe mencionar que según el último informe del Comité de las Naciones Unidas para la Eliminación de la Discriminación Racial (2014, p. 18), expresa textualmente:

El Comité nota con preocupación que los miembros de pueblos indígenas y los afroperuanos continúan siendo víctimas de una discriminación estructural, enfrentándose constantemente a la falta de oportunidades económicas, pobreza y exclusión social

El Racismo, discriminación y exclusión social también se manifiesta en casos concretos, a raíz del Terremoto del Perú del 2007 en la ciudad de Pisco, afectando a varias localidades afroperuanas del departamento de Ica, siendo según datos del Instituto Nacional de Defensa Civil (2009, p. 66):

Sismo registrado el 15 de agosto del 2007 que tuvo una magnitud de 7.9 grados en la escala de magnitud de Richter y dejó como consecuencia 576 muertos, 1,435 heridos, 64,500 viviendas totalmente destruidas e inhabitables, 219,326 personas afectadas y 431,313 personas damnificadas.

Siendo entre la mayoría de afectados, personas y comunidades afrodescendientes del sur del país, y es que, luego de 8 años y hasta la fecha no se han reconstruido gran parte de viviendas e inmuebles destruidos, demostrando una indiferencia total de la sociedad y el Estado frente a la población afrodescendiente.

A nivel social muchos afroperuanos se ven afectados por el racismo que practican tanto individuos como autoridades. Generalmente es en la escuela, en el trabajo, o en el sistema judicial donde mayormente se padecen insultos, agresiones verbales y hasta la restricción de sus derechos más elementales. Cotidianamente muchos afroperuanos y afroperuanas terminan en prisión sin contar con las mínimas garantías que les aseguren un

\footnotetext{
${ }^{2}$ Decreto Supremo № 001-MIMDES-2007.
} 
juicio justo y una adecuada defensa; en varias ocasiones la pasividad cómplice de algunas autoridades ha facilitado estos atropellos contra los derechos humanos.

En cuanto a su ubicación geográfica las y los afroperuanos por lo general residen en los barrios y lugares más pobres de las zonas urbano-marginales y agrícolas de la costa peruana, donde la falta de servicios básicos elementales afecta drásticamente los niveles y la calidad de vida. Las familias negras sufren las graves consecuencias de la desocupación, el desempleo, baja calidad educativa, el racismo y carencia de oportunidades, todo lo cual incide en el desarrollo de una débil autoestima e identidad para una gran mayoría de afrodescendientes (EL PUEBLO..., 2015).

Los hogares afroperuanos tienen un ingreso per cápita mensual menor que el promedio nacional haciéndolos ocupar estratos socioeconómicos bajos y experimentar distintos niveles de discriminación étnica que limitan la calidad y calidez de los servicios de salud, educación y empleo a los que acceden. Además, analizando las concepciones de la población afrodescendiente sobre su propia situación de pobreza, esta población se percibe víctima de una doble exclusión: debido a sus condiciones socioeconómicas y a sus características étnico-raciales ${ }^{3}$.

En el ámbito educativo, un estudio del Banco Mundial realizado en el año 2006 en Perú detalla que sólo el $27.9 \%$ de los afroperuanos tienen acceso a una educación secundaria completa y apenas el 1.9\% de los afroperuanos tienen acceso a una educación superior, La mala calidad de la educación en las zonas con presencia afrodescendiente también es un factor que ha impedido una mejor preparación para la población negra, por lo tanto, ha mantenido un desigual acceso a las oportunidades de empleo y desarrollo social. A este problema grave de educación que atraviesa el pueblo afroperuano se les agregala marginación económica, política y ciudadana los cuales son algunos de los más grandes problemas de las comunidades afroperuanas. Dicha situación se manifiesta en la escasez de oportunidades de empleo para las y los jóvenes, en las pésimas remuneraciones, la falta de crédito, y en la ausencia de iniciativas empresariales que aseguren su desarrollo; la pobreza también se muestra en las pésimas condiciones de vida en las zonas y comunidades urbano marginales.

3 Ver más datos en Comité De Las Naciones Unidas Para La Eliminación De La Discriminación Racial (2006). 
Ante la realidad histórica que atraviesa el pueblo afroperuano, se puede evidenciar que las políticas asistenciales del Estado no han sido orientadas principalmente a la población afroperuana, pero sí a la población de origen indígena, tanto en la ciudad de Lima como de la sierra del país, olvidándose que las y los afroperuanos están ubicados en su mayoría a lo largo de la costa del Perú, quienes forman parte de las poblaciones más pobres del país, lo cual es consecuencia de la invisibilidad que atraviesan aún las poblaciones afroperuanas y la poca capacidad de respuesta, organización e incidencia de esta población para promover proyectos de desarrollo inclusivos como también la lucha en contra del racismo y la discriminación.

Según Valdivia (2011, p. 72) “La exclusión que atraviesa la población afroperuana se ve plasmada también en los censos y encuestas nacionales, carentes de una variable étnica de auto identificación afrodescendiente desde 1940", lo que genera que no existan estadísticas oficiales de la población afroperuana, y mucho menos de la población joven afrodescendiente en el Perú; desconociéndose en cifras numéricas las verdaderas y actuales necesidades en educación, salud, trabajo, vivienda y otras.

Si esto es así con la población afroperuana en general es mucho más grave con la mujer afroperuana en particular, la cual es víctima del machismo y marginación por ser mujer y ser pobre. En la mayoría de los casos las afroperuanas tienen menor acceso a los servicios educativos y se desempeñan por lo general en oficios de muy baja remuneración lo cual contribuye al llamado proceso de Feminización de la Pobreza ${ }^{4}$.Sin embargo, al igual que las mujeres de otras poblaciones étnicas, las afroperuanas han sido un soporte fundamental de la familia negra y se han incorporado crecientemente a las organizaciones de sobrevivencia promovidas por las mujeres de campo y la ciudad, un ejemplo claro de estos lo constituyó María Elena Moyano ${ }^{5}$ quién ofrendó su vida heroicamente en el combate al terrorismo en defensa de la paz.

\footnotetext{
4 Para el Centro Internacional de la Pobreza (2008). La "feminización de la pobreza" es un concepto cuyos orígenes se remontan a los años 70 . Se popularizó a principio de los 90, especialmente mediante investigaciones de organismos de las Naciones Unidas. El concepto cuenta con varios significados, algunos de los cuales no concuerdan completamente con su noción implícita de cambio. Proponemos una definición en consonancia con varios estudios recientes en este campo: la feminización de la pobreza es un cambio en los niveles de pobreza que muestra una tendencia en contra de las mujeres o los hogares a cargo de mujeres.

${ }^{5}$ Fue una luchadora social, dirigente vecinal y feminista peruana, conocida popularmente como "Madre Coraje". En 1992, fue asesinada en Lima, por un comando de aniquilamiento del grupo terrorista Sendero Luminoso. En momentos en que el país se encontraba atacado por terrorismo, Moyano se alzó contra el
} 
Es necesario, dotar a las mujeres afroperuanas de los conocimientos y herramientas que posibiliten el entendimiento, uso y aplicación de sus derechos motivándolas a participar en los procesos políticos futuros que les garanticen el acceso a la justicia, que tengan capacidad de decisión y autonomía de tal manera que puedan participar y acceder a los espacios de toma de decisiones,fortaleciendo de esta manera al movimiento organizativo de mujeres no sólo en torno a las acciones de sobrevivencia, sino también en la defensa de sus derechos y fortalecimientos de la democracia.

En cuanto al ejercicio de sus derechos como ciudadanos según el último informe de Transparencia desarrollado en el año 2014se nota que la participación política-ciudadana organizada de las y los afroperuanos es de mala calidad y restringida. En efecto la idea misma de ciudadanía es poco conocida entre las y los afrodescendientes tanto porque muchos desconocen los derechos que la constitución y las leyes reconocen, porque la conciencia sobre sus responsabilidades ciudadanas se limita al ejercicio formal del voto; inclusive se puede notar entre los y las integrantes de las comunidades afroperuanas quienes carecen de documentación personal que acredite su existencia legal y el reconocimiento de su ciudadanía. Por lo general los y las afroperuanas participan poco de la vida política del país.

Sin embargo, se está evidenciando en estas últimas décadas, un creciente interés de las y los afroperuanos por cambiar esta situación. En particular a nivel local, un número de afroperuanos cada vez más significativo ha empezado a motivarse a participar efectivamente. Existen al respecto algunas iniciativas en organizaciones afroperuanas en el norte y sur de la costa del país que se deben fortalecer dotando a estos ciudadanos y ciudadanas de mayores conocimientos sobre sus derechos y deberes, mejorando su capacidad de participación y gestión ciudadana, promoviendo entre las mujeres un mayor conocimiento de su situación legal y social mejorando de esta manera la calidad de vida de las afroperuanas y jóvenes en su comunidad, acercando al Estado y a los partidos políticos a estos ciudadanos y ciudadanas.

Dentro del Movimiento Afroperuano existen pocas organizaciones trabajando los temas de derechos humanos y participación política-ciudadana, la mayoría de ellas son de

terror de Sendero Luminoso, por la lucha contra la pobreza y por la defensa de los derechos humanos. María Elena Moyano, también fue una de las más activas defensoras de los derechos de la mujer y de la población peruana en general. 
revalorización y promoción de los aportes culturales como la música, la danza, el arte y lo culinario, como manera de seguir incentivando la cultura negra desde todas las generaciones.

Otras organizaciones afroperuanas tienen como campo de acción la lucha contra el racismo en el país, como por ejemplo, la Asociación Negra de Defensa y Promoción de los Derechos Humanos ASONEDH (ASOCIACIÓN NEGRA DE DEFESA Y PROMOCIÓN DE LOS DERECHOS HUMANOS, 2015) con 25 años de fundación, es la institución más antigua trabajando actualmente en comunidades afroperuanas de todo el Perú, reconocidos por la incansable labor en la generación de espacios de formación a mujeres y jóvenes afroperuanos sobre identidad, liderazgo y derechos humanos, logrando organizar a muchas de las comunidades para sus decisiones internas, a la vez como una experiencia interesante, de promover el recambio generacional con la formación de Ashanti Perú la Red Peruana de Jóvenes Afrodescendientes (ASOCIACIÓN NEGRA DE DEFESA Y PROMOCIÓN DE LOS DERECHOS HUMANOS, 2015).

Es fundamental abrir un nuevo espacio para la preparación política de jóvenes afroperuanos que abarquen prácticas de políticas de inclusión social y acciones afirmativas, estrategias para la incidencia política y negociación, mecanismos de control y participación ciudadana, rendición de cuentas y gobernabilidad, ante la falta de representación política local, regional y nacional de la población afroperuana.

Las y los jóvenes afroperuanos que hasta el día de hoy no han recibido ningún tipo de política pública, ni acción afirmativa de parte del Estado para la reducción de estas brechas sociales étnico-racial son las y los llamados a la organización del nuevo movimiento juvenil afroperuano para contribuir a disminuir estos factores racistas de esclavización colonial con la generación y negociación de propuestas de inclusión social y desarrollo con identidad.

\section{El movimiento juvenil afroperuano y la participación política: desafíos desde la experiencia de Ashanti Perú - Red Peruana de Jóvenes Afrodescendientes}

Es notoria todavía la exclusión del pueblo afrodescendiente en los asuntos y la vida pública del país. Se muestra una gran ausencia de jóvenes afroperuanos ocupando cargos públicos de relevancia, y son víctimas de exclusión política puesto que no están 
considerados en las cuotas que deberían integrar las listas de los diferentes partidos y agrupaciones políticas a nivel nacional para los procesos electorales locales, como sí sucede con las mujeres, los jóvenes y las comunidades indígenas, existiendo aún desinterés por parte de los partidos políticos hacía la población afroperuana6.

Las y los jóvenes afroperuanos también son víctimas de discriminación asociadas al color de piel lo cual es un problema latente en la sociedad peruana que afecta diversos aspectos de la vida de la juventud afroperuana. Además de la discriminación racial, como lo mencionamos anteriormente las y los jóvenes atraviesan dificultades para acceder a las universidades, principalmente por la escasez de recursos, la desvalorización de una la identidad afroperuana por parte de ellos mismos y de la sociedad en su conjunto, y de las duras condiciones de trabajo, principalmente entre quienes residen en zonas rurales.

Los problemas mencionados repercuten en insuficientes propuestas e iniciativas que ayuden a incluir las demandas de las y los jóvenes afroperuanas, así como la discriminación racial y fomento de estereotipos hacia la población afroperuana, ocasionado con ello la acentuación de la invisibilización de la problemática de la juventud afroperuana, la carencia de políticas para revertir su situación mostrándose en la sostenibilidad de la pobreza, pobreza extrema y aumento de las brechas sociales.

Estos problemas son generados por la inexistente voluntad del Estado para diseñar e implementar políticas para las y los jóvenes afroperuanos, además de la invisibilidad de líderes y lideresas del movimiento afroperuano ejerciendo cargos de toma de decisiones.

Para que se resuelva este asunto de invisibilidad, exclusión y falta de oportunidades que atraviesa la población joven de las comunidades afroperuanas, como una esperanza de lucha contra los siglos de injusticias a causa del racismo y los prejuicios raciales, se debe tener oportunidades de empoderamiento y de tener las herramientas necesarias para poder garantizar el cambio a través de intervenciones en el ejercicio pleno de la participación ciudadana y política, a fin de influenciar y lograr la ocupación de puestos de toma de decisiones.

\footnotetext{
${ }^{6}$ Para más información ver Schmidt (2004) “Éxitos no anticipados: Lecciones de la experiencia peruana con las cuotas de género en los sistemas mayoritarios con listas cerradas y de Representación Proporcional (RP) con listas abiertas".
} 
Sin duda, con el último simbólico triunfo motivador de un afrodescendiente en la política, independientemente de su afiliación partidaria, como es el caso del Sr. Barack Obama re-elegido democráticamente como Presidente de la República de los Estados Unidos de Norte América, ha despertado una esperanza en las y los dirigentes afrodescendientes en apostar por un liderazgo político con identidad a todo nivel y recayendo también en las nuevas generaciones de jóvenes líderes afoperuanos y afroperuanas. A ello se le suma la reciente Resolución A60/460 de las Naciones Unidas que establece los años 2015 - 2024 como el Decenio Internacional de los Afrodescendientes "Reconocimiento, Justicia y Desarrollo" a manera que los Estados comiencen a promover políticas de inclusión social hacia esta población históricamente excluida.

Ante la ausencia histórica de la participación política de miembros representantes de la comunidad afroperuana y sobretodo de las y los jóvenes afroperuanos tanto en los espacios de toma de decisiones locales, regionales y nacionales como en los comicios electorales democráticos generales del Perú es que se ve la necesidad de institucionalizar espacios de formación política mediante el empoderamiento de las y los jóvenes afroperuanas para la promoción de un liderazgo juvenil que permita la aplicación de estrategias y prácticas de incidencia en políticas públicas, negociación política, desarrollo local con identidad e inclusión social, en coordinación con el Estado Peruano y las municipalidades locales y regionales.

Es en ese contexto que en los últimos años se ha venido desarrollando un proceso de fortalecimiento del Movimiento Nacional Afroperuano, mediante la formación y el ejercicio de los Derechos Humanos, ciudadanos y la democracia participativa de sus líderes y lideresas representantes de organizaciones afroperuanas. Ahora se confía y apuesta por el recambio generacional para que sean las y los jóvenes afrodescendientes dirigentes quienes continúen este proceso de lucha y reivindicación de derechos elevando su capacidad para reforzar estrategias para la inclusión, la ciudadanía, la democracia y la gobernabilidad en el Perú.

La indiferencia por parte de los tipos de gobiernos que atravesó el país y la corrupción de las autoridades de la localidad y de la región, ha generado que las y los afroperuanos no crean, ni apuesten por una democracia participativa, representativa, y en la política en general. Sin embargo, muchas de las y los jóvenes dirigentes afroperuanos son conscientes 
de que se necesita de un recambio político generacional, pero, lamentablemente cuentan con serias dificultades de cómo realizar ese cambio e incidir en estos espacios ciudadanos ante la falta de capacitación y la débil autoestima e identidad.

Al culminar este proceso de recambio generacional y empoderamiento, las y los jóvenes líderes elevarán su capacidad para fortalecer el Movimiento Juvenil Afroperuano. Es por ello, que tomando como referencia de las experiencias y estrategias de trabajo realizadas por las organizaciones juveniles afroperuanas, la organización Ashanti Perúla Red Peruana de Jóvenes Afrodescendientes viene realizando desde el 2004 un trabajo muy interesante en formación, empoderamiento, visibilidad e incidencia a nivel nacional.

Ashanti Perú combate el racismo, la discriminación y la pobreza afrodescendiente, mediante la participación activa y efectiva en el ejercicio pleno de la ciudadanía y los derechos humanos, por medio de la ejecución de acciones que fortalezcan la Identidad, Liderazgo, Derechos Humanos e Incidencia Política para jóvenes de localidades afroperuanas, en su mayoría rurales, además de la promoción de políticas públicas de inclusión social que empoderen a la juventud afroperuana a un verdadero desarrollo comunitario y su posicionamiento en puestos y espacios de toma de decisiones a nivel local, regional y nacional (ASOCIACIÓN NEGRA DE DEFESA Y PROMOCIÓN DE LOS DERECHOS HUMANOS, 2015).

Según Secretaria Nacional de la Juventud (2015, p. 79):

\begin{abstract}
Una de las experiencias que resultan interesantes de esta organización joven afroperuana es la elaboración e incidencia de la I Agenda Joven Afroperuana, la cual es un proceso que nació del clamor de las y los jóvenes líderes afroperuanos por tener una hoja de ruta que contenga sus demandas, reivindicaciones e iniciativas de políticas públicas inclusivas en Participación, Educación, Salud, Trabajo y Cultura hacia la juventud afroperuana.
\end{abstract}

Esta Agenda fue elaborada en el I Encuentro Metropolitano de Jóvenes Lideres Afrodescendientes realizado el año 2012 en el Museo Nacional Afroperuano en la ciudad de Lima con el objetivo además de incluirla en el Congreso Nacional de Juventudes frente a la participación de 1000 jóvenes líderes a nivel nacional convocado por el Estado peruano a través de la Secretaria Nacional de Juventud, en la misión de elaborar un Plan Nacional de Juventudes 2012-2021 rumbo al bicentenario de la Nación de carácter innovador e inclusivo. 
Fue la primera vez que una organización joven afroperuana participó en este espacio de debate y negociación de políticas públicas para juventudes y una de las estrategias fue el llevar consigo una Agenda establecida con reivindicaciones y políticas públicas inclusivas para la juventud afroperuana y el compromiso de una nueva generación de líderes con identidad consolidada logrando con ello poder negociar efectivamente en las mesas de trabajo, y a la vez incluir algunas de las demandas en el plan y la declaración final.

Cabe resaltar que esta experiencia fue meritoria del III Premio Nacional de Mejores Prácticas Ciudadanas en Jóvenes 2012 organizado por el Jurado Nacional de Elecciones (2012) obteniendo un reconocimiento público nacional, contribuyendo a la visibilización de la juventud afroperuana y sus procesos organizativos de defensa de derechos humanos y participación ciudadana en los procesos de toma de decisiones.

Otra de las experiencias de participación de la juventud afroperuana de esta organización son las realizaciones de Congresos Nacionales de Jóvenes Afroperuanos, el cual es un espacio nacional de jóvenes dirigentes afroperuanos y afroperuanas con el objetivo de analizar la situación actual de la juventud afroperuana, la coyuntura política local y nacional, el fortalecimiento de la identidad y las nuevas estrategias de participación ciudadana e incidencia de la Agenda de las y los jóvenes afroperuanos.

Cabe destacar también que esta organización realizó la Escuela de Formación de Jóvenes Lideres Afrodescendientes, el cual es un proceso de formación y empoderamiento de desarrollo de capacidades de 40 jóvenes líderes afroperuanos de organizaciones y comunidades de 04 provincias del departamento de Ica (Chincha, Ica, Pisco y Nazca) para la promoción y vigencia de los derechos humanos y democracia participativa, a través de un protagonismo juvenil que permite la aplicación de estrategias y prácticas de incidencia en políticas públicas, desarrollo local con identidad e inclusión social (ASOCIACIÓN NEGRA DE DEFESA Y PROMOCIÓN DE LOS DERECHOS HUMANOS, 2015).

Entre la estrategias del proceso de formación de Ashanti Perú se destacó el uso y aplicación de las $\mathrm{TIC}^{\prime} \mathrm{s}^{7}$ mediante la disposición de una plataforma virtual de capacitación, acercando a las juventudes afrodescendientes a estas nuevas herramientas 2.0 generando procesos innovadores de participación y empoderamiento, resaltando particularmente la participación de la mujer joven afroperuana.

\footnotetext{
${ }^{7}$ Tecnologías de la Información y Comunicación.
} 
Como otras de las estrategias, se evidencia la organización de réplicas locales de capacitación por parte de las y los jóvenes participantes, ejerciendo liderazgo, planificación y organización, a la vez de acercar a la comunidad en estos temas necesarios para su desarrollo.

Esta experiencia contribuyo significativamente al fortalecimiento de las habilidades y capacidades en liderazgo de muchos jóvenes afroperuanos y afroperuanas, llamando con ello la atención de dirigentes de partidos políticos locales a tal punto de involucrar a algunos y algunas jóvenes afroperuanas participantes como candidatos a regidores en las últimas Elecciones Regionales y Municipales del 2014 con una propuesta de recambio generación a la gestión política y propuestas de inclusión social.

Como uno de los resultados de este proceso Ross Lujan Carhuayo, mujer joven afroperuana de 19 años de edad, pudo ser elegida democráticamente en su distrito como regidora llevando a su partido a ganar las elecciones municipales en Acarí ${ }^{8}$.

\section{Conclusiones}

La población afrodescendiente en el Perú es un sector de la población peruana que sigue atravesando condiciones de pobreza y procesos de racismo y discriminación, y es la invisibilidad como afroperuanos y afroperuanas, la vulneración de derechos sociales, económicos, políticos los que no garantizan su desarrollo y el fortalecimiento de la identidad.

El Estado Peruano atraviesa por una ausencia histórica de acciones afirmativas y políticas de inclusión hacia la población afroperuana, no estipulando la igualdad de derechos de esta población frente a las otras poblaciones como las andinas y amazónicas, excluyendo a esta población en sus planes, programas y políticas públicas y especialmente a la juventud afroperuana, no cerrando con ello las brechas de inequidades sociales que existen.

El Estado Peruano no cuenta con cifras estadísticas oficiales que indiquen el porcentaje de jóvenes afrodescendientes en el país, sus necesidades y condiciones de vida, 
que permitan el reconocimiento y la aplicación de políticas públicas en beneficio de la juventud afroperuana.

Es importante para la continuidad y el sostenimiento de la lucha del Movimiento Nacional Afroperuano, apostar por el recambio generacional mediante la formación de nuevos líderes para continuar los procesos de defensa de derechos humanos a fin de incluir sus demandas y reivindicaciones.

El problema focal de la juventud afroperuana es la baja autoestima y desmotivación que atraviesan las y los jóvenes afroperuanos para involucrarse en espacios políticos. Este problema focal se refleja a través carencia de espacios que promuevan la inclusión de las y los jóvenes afroperuanos en el desarrollo local, la débil conciencia sobre sus derechos, deberes y libertades. Se debe promover la participación de las y los jóvenes afroperuanos en los espacios de toma de decisiones de su comunidad,

La participación política del movimiento juvenil afroperuano para la lucha en contra del racismo y la discriminación debe involucrar programas de empoderamiento y formación de jóvenes líderes afroperuanos y afroperuanas, así como la participación mediante la incidencia de una Agenda consolidada para la generación de políticas públicas de inclusión social hacia un verdadero desarrollo comunitario.

La experiencia de la Escuela de Formación de Jóvenes Lideres Afrodescendientes de la organización Ashanti Perú demuestra un fuerte espacio de formación política-ciudadana de jóvenes afroperuanos para el empoderamiento y el conocimiento de sus derechos y libertades encaminando a la incidencia política de las demandas de las poblaciones afrodescendientes, especialmente de las y los jóvenes afroperuanos.

Los espacios de participación generados por Ashanti Perú incentivan la defensa de los derechos humanos de las juventudes afroperuanas despertando el compromiso y el reforzamiento de la identidad a fin de hacerle frente a la exclusión y la pobreza.

\section{Referencias}

AGUIRRE, C. Silencios y ecos: la historia y el legado de la abolición de la esclavitud en Haití y Perú. In: Seminario Internacional sobre la Abolición de la esclavitud y SUS procesos de manimisión em El Perú, América y el Caribe. 2004, Lima. Actas... Lima: Centro de Desarrollo Étnico, CEDET, 2004. 
ASOCIACIÓN NEGRA DE DEFESA Y PROMOCIÓN DE LOS DERECHOS HUMANOS ASONEDH. Disponível em: <www.asonedhperu.org>. Acesso em: 10 out. 2015.

CENTRO INTERNACIONAL DE LA POBREZA. La feminización de la pobreza. Disponível em: <http://www.ipc-undp.org/pub/esp/IPCOnePager58.pdf>. Acesso em: 10 out. 2015

COMITÉ DE LAS NACIONES UNIDAS PARA LA ELIMINACIÓN DE LA DISCRIMINACIÓN RACIAL. Art 8 en el inciso C: motivos de preocupación y recomendación.2014. Disponível em:<http://acnudh.org/wp-content/uploads/2014/08/CERD_C_PER_CO_18-

21_18103_S.pdf>. Acesso em: 10 oct. 2014.

CONSTITUCIÓN Política del Perú. Perú, 1993.

ENCUESTA nacional continua enco. Perú. 2006.

EL PUEBLO afroperuano: principales comunidades. Disponível em: <http://www.cimarrones-peru.org/graficos/mapa_geoetnico.jpg>. Acesso em: 10 out. 2015.

INSTITUTO NACIONAL DE DEFENSA CIVIL - INDECI. Lecciones aprendidas Del sur - sismo de pisco. Lima: soluciones prácticas-ITDG, DFID.Perú. 2009.

JURADO NACIONAL DE ELECCIONES. Mejores prácticas ciudadanas de jóvenes en el Perú 2012. Lima: Dirección Nacional de Educación y Formación Cívica Ciudadana, 2012.

MARX, K. Miseria da filosofía: resposta a filosofía da miseria de PROUDHON. São Paulo: Editora Centauro, 2001.

ORGANIZACIÓN DE LOS ESTADOS AMERICANOS. Estándares de protección de afrodescendientes en el sistema interamericano. USA: OAS Cataloging-in-Publication Data OEA Documentos Oficiales,2011.

SCHMIDT, G. Éxitos no anticipados: lecciones de la experiencia peruana con las cuotas de género en los sistemas mayoritarios con listas cerradas y de Representación Proporcional con listas abiertas. Informe de Taller. Lima: International Idea, 2004.

SECRETARIA NACIONAL DE LA JUVENTUD. Juventud afroperuana: derechos humanos, participación ciudadana e incidencia política, 10 años de Ashanti Perú. Lima, 2015.

VALDIVIA, N. El uso de categorías étnico/raciales en censos y encuestas en el Perú: balance y aportes para una discusión. Lima: Grade, 2011. (Documento de Investigación, $60)$. 